\title{
Unexpected occurrence of adrenal Cushing's syndrome in a patient with systemic lupus erythematosus
}

\author{
Hypertension Research (2011) 34, 662-663; doi:10.1038/hr.2011.13; published online 17 February 2011
}

Complication of non-iatrogenic Cushing's syndrome in patients with autoimmune diseases is very rare. We report an interesting case of systemic lupus erythematosus (SLE) accompanied by Cushing's syndrome due to bilateral adrenal adenomas. A 34-year-old Japanese woman had been treated with $5 \mathrm{mg}$ per day of prednisolone and $150 \mathrm{mg}$ per day of mizoribine for lupus since the age of 13 years. When the patient was diagnosed as having SLE, she was positive for anti-nuclear and anti-double-stranded DNA antibodies with reduced levels of serum compliments. The patient's lupus activity remained stable for 4 years. However, she gradually became aware of general fatigue after June 2007. She had become hypertensive $(140 / 110 \mathrm{~mm} \mathrm{Hg})$ with the development of moon face, truncal obesity (weight gain of $4 \mathrm{~kg}$ in a 2-year period), reddish striae on her abdomen and skin atrophy when admitted in December 2007. Her hypertension was controlled by medication with nifedipine $(20 \mathrm{mg})$, spironolactone $(50 \mathrm{mg})$ and candesartan $(8 \mathrm{mg})$. Laboratory data showed moderate leukocytosis $\left(9480 \mu \mathrm{l}^{-1}\right)$, hypokalemia $\left(3.0 \mathrm{mEql}^{-1}\right)$ and increased lactate dehydrogenase $\left(525 \mathrm{IU}^{-1}\right)$, whereas anti-nuclear and anti-DNA antibodies and C-reactive protein were negative and serum complement levels were within normal limits. Abdominal computed tomography revealed bilateral adrenal tumors (Figure 1a) and a left renal nodule that was subsequently diagnosed as angiomyolipoma by biopsy. The right adrenal tumor was $28 \mathrm{~mm}$ in diameter and detected as a homogeneously low-density mass by computed tomography, whereas the left adrenal nodule was $13 \mathrm{~mm}$ in diameter with low density. Adrenal scintigraphy with ${ }^{131}$ I-adosterol revealed predominant hyperfunction of the right adrenal gland (Figure 1b). Plasma ACTH level was undetectably low $\left(<5 \mathrm{pg} \mathrm{ml}^{-1}\right)$, urinary free cortisol excretion was highly increased $(640 \mu \mathrm{g}$ per day) and cortisol secretion was excessive (25-30 $\left.\mu \mathrm{g} \mathrm{dl}^{-1}\right)$ without normal circadian fluctuation, which was not suppressed $\left(>24 \mu \mathrm{g} \mathrm{dl}^{-1}\right)$ by $1 \mathrm{mg}$ dexamethasone administration. On the basis of the clinical diagnosis of Cushing's syndrome predominantly caused by the right adrenal tumor, laparoscopic right adrenalectomy was performed on 11 March 2008. The resected adrenal tumor was pathologically confirmed to be adrenal adenoma without neoplastic or hyperplastic findings (Figures $1 \mathrm{c}$ and d). Immediately after the surgery, oral dose of prednisolone was increased to $15 \mathrm{mg}$ per day for 6 months and then gradually reduced to the initial dose of $5 \mathrm{mg}$ to prevent rebound of lupus activity. The doses of antihypertensive agents were also reduced postoperatively, and then only $4 \mathrm{mg}$ of candesartan was continued. No clinical or biochemical recurrence of lupus had been detected with subnormal blood pressure for 18 months; nevertheless, she again became aware of gradual weight gain, hypertension $(140 / 100 \mathrm{~mm} \mathrm{Hg})$, leg edema and central obesity with moon face. Of note, follow-up computed tomography showed that her residual left adrenal tumor had enlarged to $25 \mathrm{~mm}$ (Figure 1e). In contrast to the continuous suppression of ACTH levels $\left(<5 \mathrm{pg} \mathrm{ml}^{-1}\right)$, serum cotisol levels were gradually elevated (8.9-21 $\mu \mathrm{g} \mathrm{dl}^{-1}$ over a period of 18 months) with increased urinary free cortisol excretion $(522 \mu \mathrm{g}$ per day). Cortisol secretion was not suppressed by administration of $1 \mathrm{mg}$ dexamethasone $\left(>21 \mu \mathrm{g} \mathrm{dl}^{-1}\right)$. ${ }^{131}$ I-adosterol scintigraphy exhibited a high uptake in the left adrenal region without any accumulation in the right adrenal region (Figure 1f). Owing to the recurrence of Cushing's syndrome caused by enlargement of the remnant adrenal tumor, partial adrenalectomy of the residual tumor was performed on 3
December 2009. To preserve the remaining adrenal function, the adjacent normal adrenal tissue was not resected out. The left adrenal tumor was diagnosed as adrenal adenoma without other specific findings (Figures $1 \mathrm{~g}$ and h). After tumor removal, the patient received replacement therapy with $20 \mathrm{mg}$ per day of hydrocortisone in addition to $5 \mathrm{mg}$ per day of prednisolone, and her circadian cortisol levels were stabilized without recurrence of SLE.

Cases of Cushing's syndrome due to bilateral adrenal adenomas have rarely been reported, ${ }^{1-3}$ and these cases are clinically and pathologically distinct from adrenal hyperplasia such as ACTH-independent macronodular adrenal hyperplasia. A rapid enlargement of the residual adrenal adenoma was detected after unilateral adrenalectomy. As plasma ACTH levels remained suppressed by glucocorticoid replacement after the first surgery, an ACTH-independent mechanism would have been involved in the process by which Cushing's syndrome recurred. Furthermore, complications with SLE and Cushing's syndrome are extremely rare and only two cases have been reported to date. Those patients were diagnosed as having Cushing's syndrome owing to a unilateral adrenal tumor. ${ }^{4,5}$ In one patient, a 51-year-old women, steroid therapy for 5 years had been ceased for 8 years when adrenal Cushing was diagnosed. In the other patient, a 43-year-old women, lupus was controlled with a small amount $(5 \mathrm{mg})$ of prednisolone when Cushing's syndrome was diagnosed. The latter patient required intensive steroid therapy owing to recurrence of lupus activity such as hemolytic anemia and polyarthralgia after adrenalectomy. ${ }^{4}$ Glucocorticoid excess due to hypersecretion of endogenous cortisol may be favorable for suppressing activation of the concomitant autoimmunity. However, 

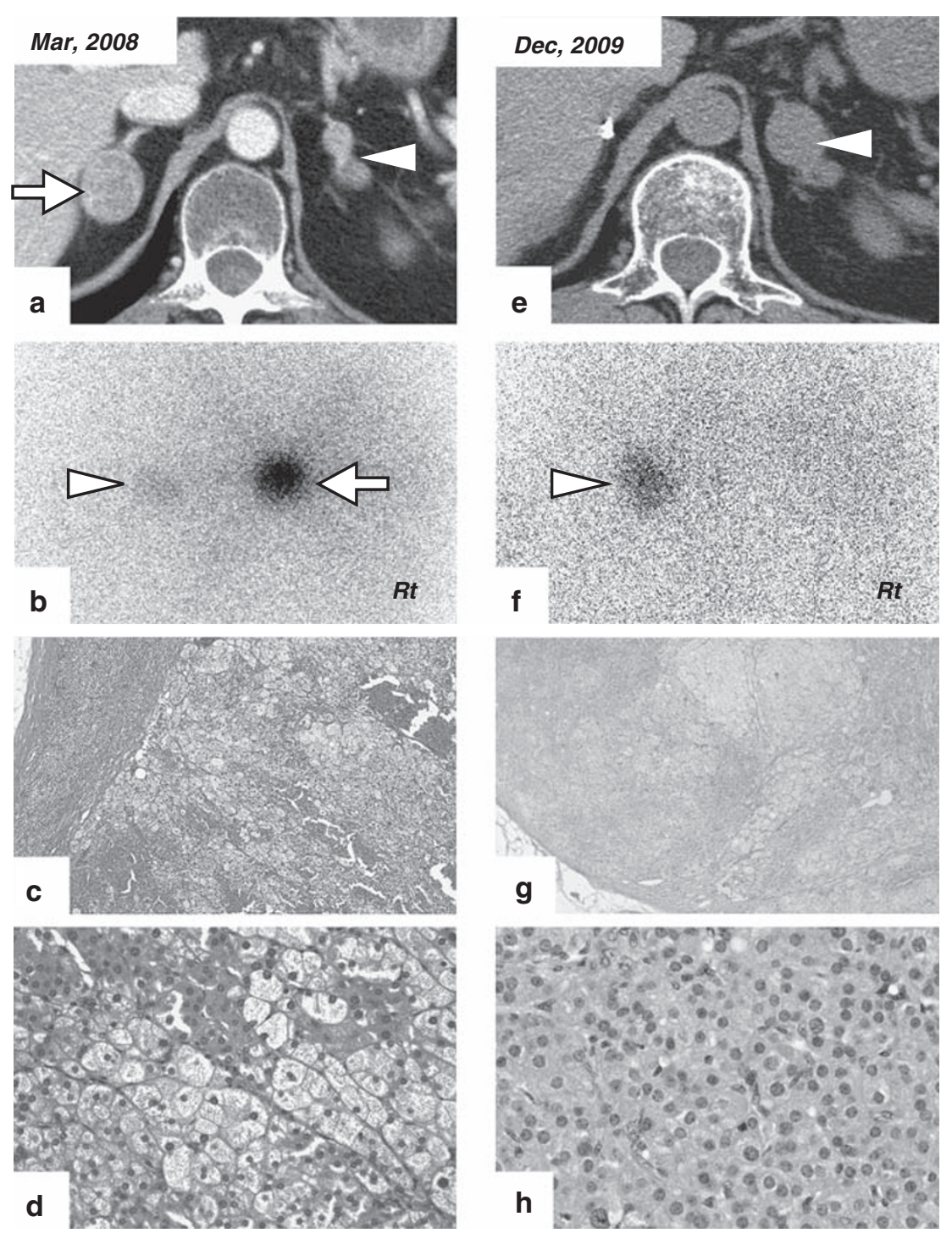

Figure 1 Characteristics of bilateral adrenal adenomas. (a, e) Computed tomography (CT) scan, (b, f) ${ }^{131}$ I-adosterol scintigraphy and (c, d, $\mathbf{g}$ and $\mathbf{h}$ ) pathological findings of the resected adrenal tumors by hematoxylin-eosin staining (c and $\mathbf{g}, \times 40 ; \mathbf{d}$ and $\mathbf{h}, \times 400$ ). Arrows indicate right adrenal adenoma and arrowheads show left adrenal adenoma.

unbalanced and excess hypercortisolemia will have various adverse effects in patients with autoimmune disorders.

The present patient is the first case of SLE complicated with Cushing's syndrome caused by bilateral adrenal adenomas. It is clinically difficult to distinguish primary Cushing's syndrome of pituitary or adrenal origin from the so-called iatrogenic Cushing's status caused by exogenously administered glucocorticoids. Therefore, it is important to evaluate endogenous glucocorticoid secretion in patients who had been treated with glucocorticoids if Cushing's signs including hypertension become rapidly overt or the activities of autoimmune diseases are unexpectedly silent or latent under the small dosage of administered immuno-suppressants.
Akihiro Katayama $^{1}$, Fumio Otsuka ${ }^{1}$, Katsuyuki Tanabe ${ }^{1}$, Naoko Tsukamoto ${ }^{1}$, Ryutaro Yamanaka ${ }^{1}$, Yoshinori Matsumoto ${ }^{1}$, Yasutomo Nasu ${ }^{2}$ and Hirofumi Makino ${ }^{1}$

${ }^{1}$ Department of Medicine and Clinical Sciences, Okayama University Graduate School of Medicine, Dentistry and Pharmaceutical Sciences, Endocrine Center of Okayama University Hospital, Kitaku,

Okayama, Japan; and ${ }^{2}$ Department of Urology, Okayama University Graduate School of Medicine, Dentistry and Pharmaceutical Sciences, Kitaku, Okayama, Japan

E-mail: fumiotsu@md.okayama-u.ac.jp
1 Tung SC, Wang PW, Huang TL, Lee WC, Chen WJ. Bilateral adrenocortical adenomas causing ACTH-independent Cushing's syndrome at different periods: a case report and discussion of corticosteroid replacement therapy following bilateral adrenalectomy. J Endocrinol Invest 2004; 27: 375-379.

2 Nomura K, Saito H, Aiba M, lihara M, Obara T, Takano K. Cushing's syndrome due to bilateral adrenocortical adenomas with unique histological features. Endocr J 2003; 50: 155-162.

3 Desai N, Kapoor A, Singh BK, Liu J. Bilateral adrenal adenomas and persistent leukocytosis: a unique case of Cushing's syndrome. Am J Med 2006; 119: e3-e5.

4 Arima K, Higuchi M, Yoshizawa S, Horiuchi T, Nagasawa K, Nakashima H, Taniguchi M, Niho Y. Improvement of systemic lupus erythematosus activity by the association of delayed onset Cushing's syndrome. J Rheumatol 1998; 25: 2456-2458.

5 Shimizu M, Kawata M, Okada T, Yuu H, Kurahashi T, Yamanaka K, Umezu K. Concomitant Cushing's syndrome due to adrenal adenoma in a patient with systemic lupus erythematosus. Intern Med 2002; 41: 1044-1046. 